High Energy Phenomena in Relativistic Outflows (HEPRO IV)

International Journal of Modern Physics: Conference Series

Vol. 28 (2014) 1460177 (6 pages)

(C) The Authors

DOI: $10.1142 / \mathrm{S} 201019451460177 \mathrm{X}$

\title{
BL LAC POPULATION STUDY AT HIGH ENERGIES*
}

\author{
LUCIE GÉRARD \\ Deutsches Elektronen-Synchrotron (DESY), \\ Platanenallee 6, 15738 Zeuthen, Germany \\ lucie.gerard@desy.de \\ GILLES HENRI \\ Institut de Planétologie et d 'Astrophysique de Grenoble, \\ BP 5338041 Grenoble Cedex 9, France \\ gilles.henri@obs.ujf-grenoble.fr \\ SANTIAGO PITA ${ }^{\dagger}$ and MICHAEL PUNCH ${ }^{\ddagger}$ \\ Université Denis Diderot, Laboratoire AstroParticule Cosmologie (APC), \\ 10 rue Alice Domon et Léomie Duquet, 75205 Paris, France \\ ${ }^{\dagger}$ pita@apc.univ-paris7.fr \\ ${ }^{\ddagger}$ punch@apc.univ-paris\%.fr
}

Received 31 October 2013

Revised 21 November 2013

Published 21 March 2014

In the framework of Active Galactic Nuclei (AGN) unification, BL Lacs and their parent population would share the same intrinsic characteristics, the observational differences being due to the orientation of the relativistic jet compared to the line of sight. BL Lacs would be the objects whose jet is oriented towards us, their emission being amplified by the relativistic Doppler boosting. Constraints arising from fast variability and/or large optical depth to pair production commonly imply large Lorentz factors. The growing number of BL Lacs detected at $\mathrm{HE}(>100 \mathrm{MeV})$ and VHE $(>100 \mathrm{GeV})$ is a challenge for this unification scheme. Indeed, the high values of Doppler factor needed in the simplest radiative model to explain the emission of these sources imply a large density for the parent population. A possible solution to this Doppler factor crisis lies in considering different geometries for the jet. In this study, we use the BL Lacs detected at HE and VHE to investigate the intrinsic properties of the associated parent population. Using the results presented in Fermi's second AGN catalog and performing MC simulations of the parent population, we constrain the jet parameters: its intrinsic luminosity, Lorentz factor and geometric opening angle. The simulated density of parent population and

*This is an Open Access article published by World Scientific Publishing Company. It is distributed under the terms of the Creative Commons Attribution 3.0 (CC-BY) License. Further distribution of this work is permitted, provided the original work is properly cited. 
Doppler factors of the objects detectable at HE within this population are presented according to the jet parameters.

Keywords: AGN; BL Lacs; FRI; unification; jet; high energy.

PACS Numbers: 98.54.Cm, 98.54.Gr, 98.58.Fd

\section{Introduction}

BL Lacs are blazars, AGN whose jet is believed to be directed towards us. Their observed emission is thus amplified by the relativistic Doppler boost. They are the most energetic known class of AGN. At Very High Energy (VHE, > $100 \mathrm{GeV}$ ), $\sim 50$ blazars have now been detected with the current generation of Atmospheric Cherenkov Telescopes (ACTs), most of them being BL Lacs. At High Energies (HE, between $100 \mathrm{MeV}$ and $100 \mathrm{GeV}$ ), more than a thousand blazars have been reported in Fermi's $2^{\text {nd }}$ AGN catalog, including $~ 400$ identified BL Lac type AGN.

In the framework of AGN unification, blazars are associated with a parent population. Due to the orientation of the jet compared to the observer line of sight the blazar and the parent population appear different but they intrinsically share the same characteristics. Fanaroff Riley type I (FRI) radio galaxies are the parent population usually associated with BL Lacs, on the basis of radio, optical and X-ray observations (see Ref. 1 and references therein). Here we will consider this association in the light of the BL Lacs detected at HE and VHE energies.

At these energies, considering the constraints arising from fast variability and/or large optical depth to pair production, simple radiative models need high Doppler factors $(30-50)$ to explain the emission of blazars. The Doppler factor is defined as $\delta=[\Gamma(1-\beta \cos \theta)]^{-1}$, where $\Gamma$ is the Lorentz factor, $\theta$ the jet viewing angle and $\beta=v / c$ with $v$ the jet velocity. The required high values of Doppler factors thus imply high velocity (Lorentz factor $\Gamma$ ) jets oriented close to the line of sight. Since large $\Gamma$ lead to small radiative opening angles for the jet, blazars are statistically rare objects. The large population of BL Lacs detected at extreme energies implies a much larger parent population, composed of the same type of AGN but with their jets directed away from us. With such high values of $\delta$ the density of the parent population of BL Lacs could be larger than that of FRI, their common counterpart. A possible way out of this Doppler factor crisis $^{2}$ is to consider a jet with a geometrical opening angle, increasing the probability of seeing an object within the jet's cone.

In this study, the population of BL Lacs emitting at HE is considered to constrain the intrinsic properties of these objects. The focus is on investigating the jet characteristics, particularly its geometric opening angle and how this characteristic scales with the density of the parent population. Extensive Monte Carlo simulations of the parent population are performed and the distribution of objects detectable by current instruments is compared to data, mainly Fermi's $2^{\text {nd }}$ AGN catalog. ${ }^{3}$ 


\section{Presentation of the Study}

Unlike the sample of AGN detected at VHE - still statistically limited and suffering from neither being complete nor unbiased - the sample of AGN detected under sky survey mode of observation and reported in Fermi's $2^{\text {nd }}$ AGN catalog is well suited for population study. From this catalog, the AGN labeled as BL Lacs, belonging to the clean sample and with known redshift (z) are selected. Only the objects with $z<0.5$ are considered. This should reduce the bias due to ignoring the objects with unknown redshift and the BL Lacs evolution. A sample of 130 BL Lacs is thus obtained. The flux measured between $100 \mathrm{MeV}$ and $100 \mathrm{GeV}$, the spectral index and the redshift reported for each object in Fermi's $2^{\text {nd }}$ AGN catalog are used, as well as Fermi's sensitivity. The relation between the spectral index and the energy of the peak emission presented in Ref. 4 is also used.

In the following, a population of AGN is simulated, with a certain set of intrinsic characteristics and under some hypothesis presented below. The flux at HE is evaluated and the objects that would be detectable by Fermi are selected. Their distribution is then compared to that of the Fermi selected sample.

The spectral energy distribution of BL Lacs is characterized by a double bump structure. This study concentrates exclusively on the high energy peak of the emission - covered by Fermi and the ACTs. Looking at the emission coming from the jet, the intrinsic properties of the objects are screened by the Doppler boost. The observed emission is completely characterized by the spectral shape, which is not modified by the Doppler boost, and by the energy $\left(E_{p}\right)$ and the the apparent isotropic luminosity $\left(L_{p}\right)$ of the peak emission defined as:

$$
\begin{gathered}
E_{p}=\delta E_{p}^{i} \\
L_{p}=\delta^{p} L_{p}^{i}
\end{gathered}
$$

where the $i$ stands for intrinsic and $\delta$ is the Doppler factor.

In the simulation, AGN are taken to have two symmetrical jets with continuous outflow $(p=3$ ) and a homogeneous spatial distribution up to $z=0.5$. For the high energy peak emission of the parent population, we assume a unique empirical spectrum shape: a broken power law with curved transition derived from contemporaneous spectrum of BL Lacs measured with Fermi and ACTs. The distribution of the jet intrinsic luminosity is supposed to follow a power of index $\alpha_{L}$. A relation between $E_{p}^{i}$ and $L_{p}^{i}$ :

$$
L_{p}^{i}=e^{C}\left(E_{p}^{i}\right)^{-t}
$$

is assumed with $t \sim 0.45$. And we assume a power law distribution for Lorentz factors, with index $\alpha_{\Gamma}$ and minimum value $\Gamma_{\min }$. The jet characteristics, also free parameters of the simulation, are: $\alpha_{\Gamma}, \Gamma_{\min }, \alpha_{L}, C$ and the geometric opening angle of the jet $\theta_{\text {geo }}$.

For each object of the population, the intrinsic characteristics $\left(\Gamma, L_{p}^{i}, E_{p}^{i}\right)$, the redshift, and the orientation of the jets compared to the line of sight are simulated. 

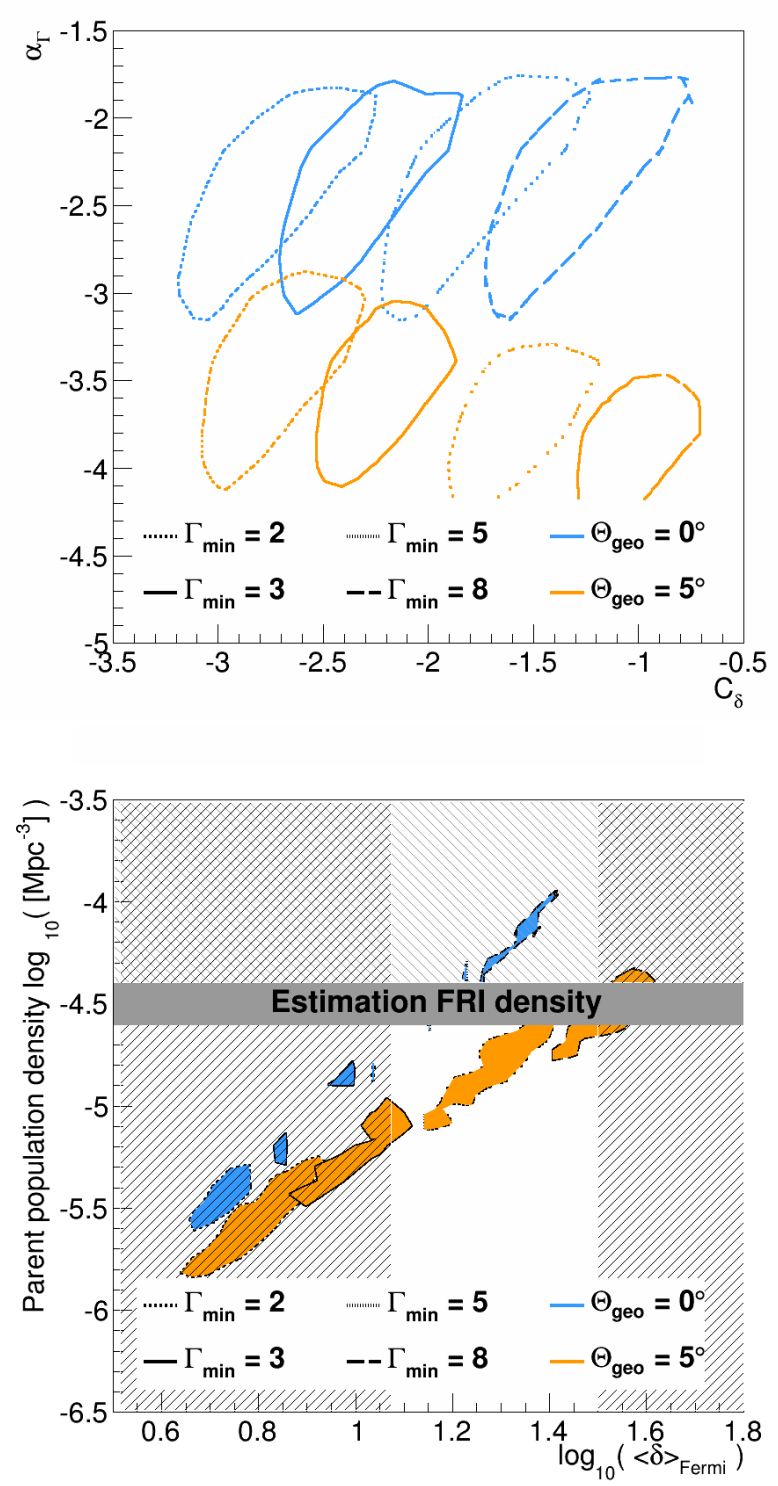

Fig. 1. Constraints on the jet characteristics. Top: Within the contours are the simulated jet characteristics for which the $E_{p}-L_{p}$ objects distribution is compatible within $3 \sigma$ with the distribution of BL Lacs detected by Fermi. For a given $C, C_{\delta}=\log \left(\delta_{\min }\right)$ where $\delta_{\min }$ is the minimal Doppler factor needed for an object at $z=0.001$ to be detectable by Fermi. Bottom: Colored region are the densities of parent population simulated with the jet parameters contained in the $3 \sigma$ contours of the top figure. $\langle\delta\rangle_{F e r m i}$ is the mean Doppler factor of the AGN found to be detectable by Fermi. The usual range of $\delta$ for BL Lacs in Synchrotron Self Compton models lies in the un-hatched region. 
The Doppler boost is applied to the emission. Using the empirical spectral shape, the fluxes in the energy ranges covered by Fermi and by the ACTs are calculated. The absorption due to the extragalactic background light is taken into account considering Franceschini's model. ${ }^{5}$ The simulations are performed for different jet characteristics. The number of objects found to be detectable by Fermi (or by the ACTs), their distribution in the $E_{p}-L_{p}$ plane and their Doppler factor are the output of the simulations.

\section{Results}

The simulated distribution — in the $E_{p}-L_{p}$ plane — of AGN detectable by Fermi is compared to the experimental one using a likelihood calculation. The intrinsic luminosity distribution of the parent population is found consistent with a power law of index $\alpha_{L}=-2.2 \pm 0.4$. The range of simulated jet characteristics compatible with Fermi data is represented on Fig.1-Top. Jets with different geometric opening angle and different $\Gamma_{\text {min }}$ can reproduce the data. In order to further constrain those parameters other observables are extracted from the simulations.

The simulated density of the parent population is calculated, normalizing the number of objects detectable at HE to the number of BL Lacs detected by Fermi. This density is a lower limit. Indeed, it only considers the objects above the minimal intrinsic luminosity below which AGN emission, regardless of their boost, could not be detected by current instruments. This lower limit is bound to increase as the sensitivity of the instruments gets better with time. Figure 1-Bottom represents the density of parent population as a function of the mean $\delta$ of the AGN found to be detectable by Fermi. The filled regions represent densities compatible with Fermi data. The simulated parent population density cannot be higher than that of FRI type AGN, if those AGN are associated with BL Lacs. The density of FRI can be roughly estimated supposing they represent $\sim 10 \%$ of the elliptical galaxies. As illustrated on Fig.1-Bottom, within this limit it seems difficult to reach high $\delta$ without considering a geometric opening angle for the jet.

The number of AGN found to be detectable at VHE does not further constrain the results as it is compatible with the number of BL Lacs detected by the current generation of ACTs.

\section{Discussion}

These simulations show that the distribution of BL Lacs detected by Fermi can be reproduced under the hypothesis of the intrinsic relation between the luminosity and energy of the emission peak defined in Eq. (1). The results are shown here for $t=0.45$. Limits on the range of $t$ values compatible with Fermi data as well as the impact of this value on the results are being investigated.

It has been argued $^{6}$ that most of objects with unknown redshift could have $z>0.5$. Recent measurements ${ }^{7}$ involving different methods show that roughly half of the BL Lacs with unknown redshift could have a redshift lower limit below 0.5. 
Limiting the study to $z<0.5$ does not shield from the influence of AGN without a known redshift. One can argue that the distribution of AGN is not dependent of the knowledge of their redshift, still, a careful evaluation of the possible bias is needed.

\section{Conclusion}

Within the framework of AGN unification, and assuming simple hypotheses for the parent population, we investigate the jet properties of BL Lacs using Fermi's $2^{\text {nd }}$ AGN catalog and extensive Monte Carlo simulations. The intrinsic luminosity distribution and jet velocity of BL Lacs emitters at high energy are constrained. Lower limits on the density of their parent population are derived. For Doppler factors higher than 20, it appears difficult to consider FRI as the parent population of BL Lacs without a geometric opening of the jet.

\section{Acknowledgments}

L.G. acknowledges support through the Young Investigators Program of the Helmholtz Association.

\section{References}

1. C.M. Urry et al., ApJ 382, 501-507 (1991).

2. G. Henri and L. Saugé, ApJ 640, 185-195 (2006).

3. M. Ackermann et al., ApJ 743 (2011).

4. A. A. Abdo et al., ApJ 716, 30-70 (2010).

5. A.Franceschini et al., A\&A 487, 837-852 (2008).

6. A. A. Abdo et al., ApJ 715, 429-457 (2010).

7. Shaw M. et al., ApJ, 764135 (2013). 